\title{
TiPd Shape Memory Alloy Studied by PAC Method
}

\author{
A. KULiŃSKA* AND P. WODNIECKI \\ Institute of Nuclear Physics PAN, E. Radzikowskiego 152, 31-342 Kraków, Poland
}

\begin{abstract}
Perturbed angular correlation (PAC) method was applied to study the martensitic phase transition of the TiPd shape memory alloy doped with In, Hf, and Zr. The hyperfine interaction parameters and their concentration and temperature dependences for ${ }^{111} \mathrm{In} /{ }^{111} \mathrm{Cd}$ and ${ }^{181} \mathrm{Hf} /{ }^{181} \mathrm{Ta}$ probe atoms were determined. The influence of ternary element additions on the electric field gradients at $\mathrm{Pd}$ site was evidenced. A decrease of the martensite start temperature $\left(M_{\mathrm{S}}\right)$ and an increase of the width of the hysteresis loop $\left(T_{\mathrm{H}}\right)$ with an increasing concentration of the impurities in TiPd alloy was observed. These relationships are much stronger for In and $\mathrm{Zr}$, than for Hf admixture.
\end{abstract}

DOI: 10.12693/APhysPolA.125.936

PACS: 81.30.kf, 62.20.fg

\section{Introduction}

Shape memory alloys (SMA) are a unique class of metal alloys of very important technological applications, including mechanical actuator devices, electronics, and medical stents. The shape memory effect is related to a reversible martensitic (diffusionless) phase transformation, which can be induced by applied fields, temperature, or both, as well as the mechanical properties of materials and, therefore, can be well controlled. The addition of a small amount of ternary element is one of the effective methods for changing and controlling the martensitic start temperature $\left(M_{\mathrm{S}}\right)$. The transformation exhibits hysteresis, in which the transformations on heating and on cooling do not overlap. The shape and the width $\left(T_{\mathrm{H}}\right)$ of the hysteresis loop also depend on doped atoms. In the last few decades, the Ti-based alloys have been a subject of intensive experimental and theoretical investigations. In many practical applications of SMAs, the most commonly used material is NiTi. Otsuka and Ren [1] give the extensive review of the Ti-Ni-based alloys. Substitution of a third element for $\mathrm{Ni}$ or $\mathrm{Ti}$ has been proved to be an effective way to increase the transformation temperature [2-4].

The TiPd shape memory alloys are well-known as potential materials for high temperature applications, since the intermetallic phase of TiPd undergoes a thermoelastic transformation from a simple cubic $B 2$ structure to an orthorhombic $B 19$ structure around $800 \mathrm{~K}$ [5]. The unit cell of $B 2$ phase (space group $P m \overline{3} m$ ) is characterized by one $\mathrm{Pd}$ site labeled $1 \mathrm{a}$ and one $1 \mathrm{~b}$ Ti site, both having cubic symmetry. In the unit cell of $B 19$ phase (space group Pmma), the Pd atoms occupy 2e sites, while the Ti atoms are situated in $2 \mathrm{f}$ positions $[6,7]$. These sites have a lower symmetry and, therefore, for each of them one can expect the non-axially symmetric electric field gradient (EFG). Since the martensitic transformation is a type of non-diffusive structure phase transformation,

*corresponding author; e-mail: Agnieszka.Kulinska@ifj.edu.pl involving a change in the shape of the unit cell together with the atomic scale displacements of atom positions in the lattice, the application of the perturbed angular correlation (PAC) method, sensitive for the changes of microstructure, is justified.

\section{Experimental details \\ 2.1. Sample preparation}

The TiPd alloys were prepared by multiple arc melting under argon atmosphere of the proper amounts of high purity components. The nominal composition of alloy was determined by the masses of components, where a minor mass loss appearing during the melting procedure was attributed to evaporation of the most volatile element. The four sets of samples were prepared: pure without admixture, doped with hafnium, indium and zirconium admixture of different content. The maximum concentrations of impurity added to the compounds reached: $6.47(3)$ at.\% Hf, 7.48(3) at.\% In, and 2.33(3) at.\% Zr. The pure sample and these with additional $\mathrm{In}$ and $\mathrm{Zr}$ atoms were measured using ${ }^{111} \mathrm{In} /{ }^{111} \mathrm{Cd}$ probes, while the samples with $\mathrm{Hf}$ using ${ }^{181} \mathrm{Hf} /{ }^{181} \mathrm{Ta}$ probes.

The ${ }^{111} \mathrm{In} /{ }^{111} \mathrm{Cd}$ probes at energy of $400 \mathrm{keV}$ were implanted into the slice-shaped samples at room temperature by means of the Göttingen heavy ion implanter IONAS [8]. After implantation, heat treatment at $1073 \mathrm{~K}$ for a few hours was applied in order to remove the irradiation damages. The ${ }^{181} \mathrm{Hf} /{ }^{181} \mathrm{Ta}$ probes were obtained by neutron irradiation of natural hafnium in the pile of the MARIA reactor at Świerk at a flux of about $10^{14}$ neutrons $/\left(\mathrm{cm}^{2} \mathrm{~s}\right)$. The ${ }^{181} \mathrm{Hf} /{ }^{181} \mathrm{Ta} \mathrm{PAC}$ probes were introduced by additional arc melting of the sample with a few milligrams of the radioactive hafnium. After fast cooling down in the arc oven, the samples were annealed in evacuated and sealed quartz tubes at $1273 \mathrm{~K}$ for $1 \mathrm{~h}$ to ensure the homogenization. The X-ray diffraction measurements were performed at room temperature with $\mathrm{Cu} K_{\alpha}$ radiation to check the stoichiometry and crystallographic structure of the samples. The analysis of the XRD spectra confirmed formation of the low temperature orthorhombic TiPd phase with B19 structure without any admixture of the other TiPd compounds for the 
samples up to about 7 at.\% of impurity. This indicates that a small addition of ternary element does not influence formation of the $B 19$ single phase in the TiPd alloy.

\subsection{Perturbed $\gamma-\gamma$ angular correlation method}

The perturbed $\gamma-\gamma$ angular correlation method is one of the nuclear methods in solid state physics appropriate for studying the structural and electronic properties of probe atoms in solids. The PAC method has been reviewed extensively in the literature $[9,10]$. EFG acting on the probe nuclei can be characterized by only two parameters: the asymmetry parameter $\eta=\left(V_{x x}-V_{y y}\right) / V_{z z}$, which represents the deviation from the axial symmetry $(0 \leq \eta \leq 1)$, and $V_{z z}$ - the largest EFG component in the principal axis system, which represents the strength of the EFG. The analysis of experimental $R(t)$ spectra was performed assuming the expression of the perturbation factor $G_{22}(t)[9]$ valid for the static electric hyperfine interactions

$$
\begin{aligned}
& G_{22}(t)=\sum_{i=1}^{k} f_{i} \sum_{n=0}^{3} s_{2 n}\left(\eta_{i}\right) \cos \left(g_{n}\left(\eta_{i}\right) \nu_{Q i} t\right) \\
& \quad \times \exp \left(-g_{n}\left(\eta_{i}\right) \delta_{i} t\right) .
\end{aligned}
$$

The least squares fits of the perturbation factor to the experimental data yield the fractions $f_{i}$ of probes exposed to different EFG's characterized by the quadrupole frequencies $\nu_{Q i}$ and asymmetry parameters $\eta_{i}$.

A broadening of EFG is described by the relative width $\delta_{i}$ of the Lorentzian distribution around $\nu_{Q i}$. The various observed fractions $f_{i}$ of different PAC parameters indicate the population of non-equivalent probe sites in the lattice. The amplitudes $s_{2 n}$ and the functions $g_{n}(\eta)$ can be calculated according to Ref. [11]. As no texture has been evidenced in the samples, the data were fitted with $s_{2 n}$ parameters for polycrystalline compound. The known quadrupole moment $Q=2.36(5)$ b for ${ }^{181} \mathrm{Ta}[12]$ and $Q=0.83(5)$ b for ${ }^{111} \mathrm{Cd} \mathrm{[13]} \mathrm{were} \mathrm{adopted} \mathrm{in} \mathrm{the} \mathrm{cal-}$ culation of the principal EFG component $V_{z z}$ according to $V_{z z}=h \nu Q / e Q$.

The PAC measurements were carried out in the temperature range 295-1073 $\mathrm{K}$ using the standard four $\mathrm{NaI}(\mathrm{Tl})$ - or $\mathrm{BaF}_{2}$-detector setups. In the temperature range where the martensitic transformation takes place the spectra were collected in a step of $5-10 \mathrm{~K}$ to ensure the determination of $M_{\mathrm{S}}$ temperature with good accuracy.

\section{PAC results and discussion}

\subsection{Concentration dependence on the hfi parameters}

The room temperature PAC spectra taken for the TiPd alloy with ${ }^{181} \mathrm{Ta}$ and ${ }^{111} \mathrm{Cd}$ probes can be described by the two quadrupole frequencies both with asymmetry parameters differing from zero. For the pure TiPd sample measured with ${ }^{111} \mathrm{Cd}$, about $70 \%$ of the probe atoms exhibit EFG1, described by the well defined quadrupole frequency $\nu_{Q 1}=48.8(7) \mathrm{MHz}$ and asymmetry parameter $\eta_{x}=0.34(1)$. The rest of the probes exhibit slightly smaller EFG2 $\left(\nu_{Q 2}=37(2) \mathrm{MHz}\right)$ with a larger asymmetry parameter $\left(\eta_{2}=0.83(3)\right)[14]$. For the $\mathrm{TiPd}$ with lowest hafnium concentration measured with ${ }^{181} \mathrm{Ta}$ about $90 \%$ of the probe atoms exhibit EFG1, characterized by the quadrupole frequency $\nu_{Q 1}=312(2) \mathrm{MHz}$ $\left(\eta_{1}=0.19(1)\right)$. The rest of the probes exhibit slightly larger EFG2 $\left(\nu_{2}=354(2) \mathrm{MHz}\right)$ with larger asymmetry parameter $\left(\eta_{2}=0.59(3)\right)[15]$.

TABLE I

The experimental $[14,15]$ and calculated [16] parameters of the electric field gradient at ${ }^{111} \mathrm{Cd}$ and ${ }^{181} \mathrm{Ta}$ probes in TiPd. $V_{z z}$ in units of $10^{21} \mathrm{~V} / \mathrm{m}^{2}$.

\begin{tabular}{c|c|c|c|c|c|c|c|c}
\hline \hline Probe & $\begin{array}{c}\text { cattice } \\
\text { site }\end{array}$ & $\begin{array}{c}\nu_{\mathrm{Q}}(\mathrm{RT}) \\
{[\mathrm{MHz}]}\end{array}$ & $\eta$ & $\begin{array}{c}\left|V_{z z}\right| \\
(\mathrm{RT})\end{array}$ & $\begin{array}{c}\left|V_{z z}\right|^{\text {exp }} \\
(0 \mathrm{~K})\end{array}$ & $\begin{array}{c}V_{z z}^{\text {calc }} \\
(0 \mathrm{~K})\end{array}$ & $\eta^{\text {calc }}$ & Impurity \\
\hline \multirow{2}{*}{${ }^{111} \mathrm{Cd}$} & $\mathrm{Ti} 2 f$ & $48.8(7)$ & $0.34(2)$ & $2.43(3)$ & $2.76(1)$ & 2.2 & 0.56 & pure \\
& $\mathrm{Pd} 2 e$ & $37(2)$ & $0.83(3)$ & $1.84(5)$ & & 1.9 & 0.66 & \\
\hline \multirow{2}{*}{${ }^{181} \mathrm{Ta}$} & $\mathrm{Ti} 2 f$ & $314(2)$ & $0.15(2)$ & $5.50(1)$ & $5.74(1)$ & 5.5 & 0.05 & Hf \\
& Pd $2 e$ & $354(4)$ & $0.59(3)$ & $6.20(2)$ & & -14.1 & 0.42 & 0.18 at.\%
\end{tabular}

Recently the results of the $a b$ initio calculation based on density functional theory (DFT) performed by Belošević et al. [16] for TiPd intermetallic compounds confirmed the assumption that the ${ }^{111} \mathrm{Cd}$ and ${ }^{181} \mathrm{Ta}$ substitutes mainly $\mathrm{Ti}$ atoms in the lattice (Table I). The location of the ${ }^{111} \mathrm{In} /{ }^{111} \mathrm{Cd}$ probes in the $\mathrm{Pd}$ site was also verified by these calculations. Since the measured and calculated $V_{z z}$ values at ${ }^{181} \mathrm{Ta}$ substituted $\mathrm{Pd}$ site in TiPdHf alloy do not match each other, the origin of the second EFG2 is still not clear. On the other hand, similar values of the both EFGs but the larger $\eta$ parameter and larger frequency distribution $\delta$ describing the EFG2 can indicate that the corresponding probe atoms are placed in Ti-sites but with not perfect environments, disturbed by defects and/or the presence of the other impurity atoms in the probe's neighborhood.

The PAC results obtained for TiPd alloys with the various admixtures and their concentrations gave the information about the compositional dependence of the electric field gradient acting on the probe nuclei at $\mathrm{Ti}$ and $\mathrm{Pd}$ sites. Figure 1 presents the concentration dependence of the hfi parameters characteristic for the EFG1 (solid symbols) and EFG2 (open symbols) in TiPd alloy with Hf, In, Zr admixture, obtained at room temperature.

As can be seen in the left part of Fig. 1, the value of the EFG1 corresponding to the ${ }^{111} \mathrm{Cd}$ and ${ }^{181}$ Ta probes substituting Ti atoms was almost independent of the admixture content. However, the significant growth of the EFG2 was observed up to 1 at. $\%$ of indium and zirconium. The data obtained for these two admixtures can be fitted according to the known empirical function $\nu_{Q}(x)=r[1-\exp (-c x)][17]$. The determined parameter $r$ reaches 1.43 and 1.3 for $\mathrm{In}$ and $\mathrm{Zr}$, respectively, giving the $\nu_{Q 2}^{\max }=58(1) \mathrm{MHz}$ for indium and $\nu_{Q 2}^{\max }=53(2) \mathrm{MHz}$ for the zirconium impurity. The EFG 2 measured for the samples with Hf admixture did not follow this relationship. This favors the interpretation that in this case the probes are placed at the interstitial or disordered lattice site. 


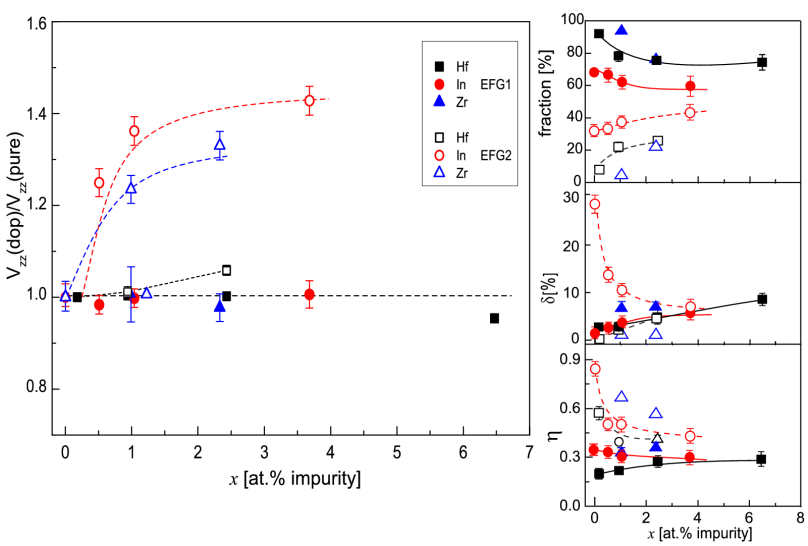

Fig. 1. Concentration dependence of the hfi parameters obtained at room temperature in TiPd alloy doped with Hf, In, and Zr.

The population of the $\mathrm{Ti}$ site decreased with increasing impurity concentration, reaching about $80 \%$ and $60 \%$ for the maximum content of $\mathrm{Hf}, \mathrm{Zr}$, and $\mathrm{In}$, respectively. Simultaneously, the width $\delta$ of the frequency distribution increased, however only small changes in asymmetry parameter $\eta$ as observed for all types of admixtures (Fig. 1, right parts). The variations of the frequency distributions and asymmetry parameters with the impurity content can be connected with the oversized probe atoms comparing to the constituent atoms of TiPd compound and with the additional impurity atoms or/and defects nearby the probes.

\subsection{Effect of ternary addition on the $M_{\mathrm{S}}$ and $T_{\mathrm{H}}$}

In order to investigate the influence of impurity and its concentration on the martensitic transformation parameters: the martensitic start temperature $M_{\mathrm{S}}$ and the width of hysteresis loop $T_{\mathrm{H}}$, the series of PAC experiments were performed for TiPd alloy doped with wide range of $\mathrm{Hf}$, In, and $\mathrm{Zr}$ admixtures. The temperature dependences of the martensite fraction obtained by the PAC are presented in Fig. 2. Only three of ten different concentrations of impurity (pure, 0.18 at.\% Hf and 1.04 at.\% In) are plotted for the clarity of the figure. The martensitic fraction reached $100 \%$ only for $x=0.18$ at. $\%$ Hf. For the higher contents of $\mathrm{Hf}$ as well as for other admixture the fraction is smaller due to the presence of defects and additional impurity atoms in the neighborhood of the probe atoms. As it is visible in Fig. 2, the drastic decrease of the $M_{\mathrm{S}}$ temperature with increasing impurity concentration $x$ was observed simultaneously with increase of the width $T_{\mathrm{H}}$.

Kawamura et al. [18] reported a similar behavior, evidenced in TiAu alloy doped with various elements. A linear decrease in the $M_{\mathrm{S}}$ temperature with increasing concentration (up to 3 mol.\% of the impurity) was observed. In these alloys, they found almost linear relationship between the $M_{\mathrm{S}}$ and $e / a$ (electron-atom ratio) values, calculated as a number of the sum of the outer $s$ - and

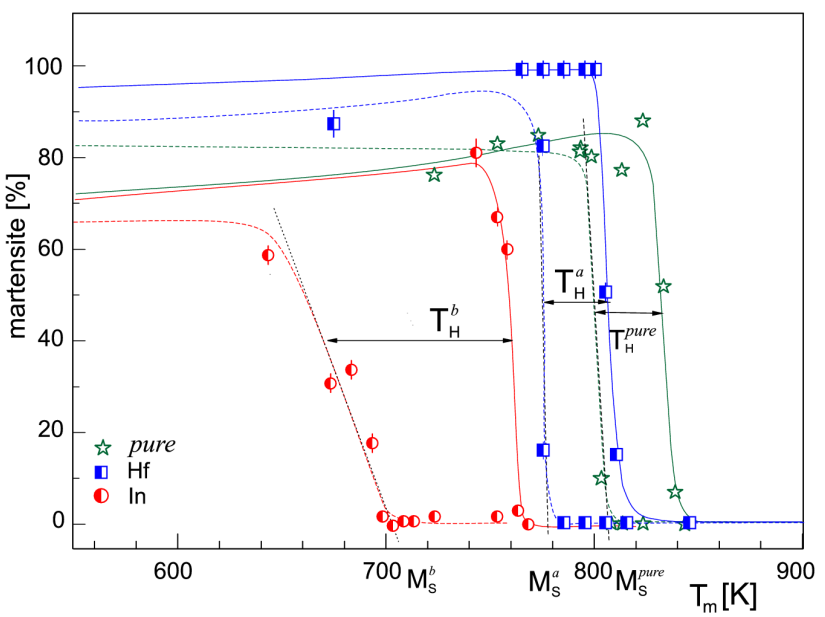

Fig. 2. Temperature dependence of the martensitic fraction in TiPd compound of selected impurity and its concentration: pure - without admixture, $a$ 0.18 at.\% Hf, $b-1.04$ at.\% In.

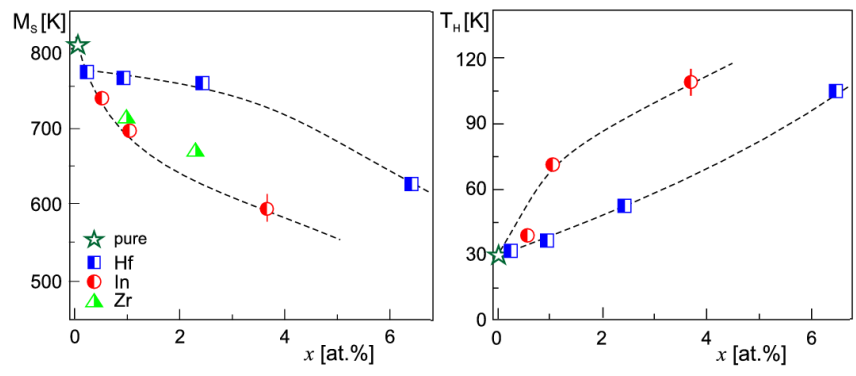

Fig. 3. Martensitic start temperature $M_{\mathrm{S}}$ and the width of the hysteresis loop $T_{\mathrm{H}}$ obtained for TiPd alloy.

$p$-electrons. Moreover, they suggest that not only the $e / a$ number, but also other factors must influence the $M_{\mathrm{S}}$.

Figure 3 presents the $M_{\mathrm{S}}$ and $T_{\mathrm{H}}$ values, obtained by PAC method as a function of the content $x$ of the third element in TiPd alloy. The strong influence of the admixture on the martensitic transformation is clearly visible. Although, these phenomena has been reported for several shape memory alloys [18-20], its origin is still not fully understood. As can be seen in Fig. 3, both the $M_{\mathrm{S}}$ and $T_{\mathrm{H}}$ concentration dependences are much stronger for In and $\mathrm{Zr}$ admixture than for Hf. It is noticeable that only for very low impurity concentration $x$ the linear relationship can be observed. The results obtained for $\mathrm{Hf}$ and In impurities reported in Refs. [14] and [15] confirmed that the $M_{\mathrm{S}}$ in TiPd depends on the $e / a$ number as well. However, for $\mathrm{Zr}$ admixture this so strong relationship was not observed.

The calculated $e / a$ number and the measured temperature $M_{\mathrm{S}}$ for the samples with impurities concentration about 1 at.\% are summarized in Table II. The $e / a$ value was calculated taking into account $f+d+s, d+s$, and $d+s+p$ electrons for $\mathrm{Hf}, \mathrm{Zr}$, and In admixtures, respectively. Our results supported the suggestion that many factors should be taken into account, when the effect of 
TABLE II

Influence of the ternary addition on the martensitic start temperature $M_{\mathrm{S}}$ observed in TiPd alloy.

\begin{tabular}{c|c|c|c}
\hline \hline Impurity & $\begin{array}{c}\text { Concentr. } x \\
\text { [at.\%] }\end{array}$ & $e / a$ & $\begin{array}{c}M_{\mathrm{S}} \\
{[\mathrm{K}]}\end{array}$ \\
\hline Hf & 0.94 & 7.129 & $767(2)$ \\
Zr & 0.99 & 7.043 & $710(2)$ \\
In & 1.04 & 7.029 & $700(2)$
\end{tabular}

ternary addition on the martensitic transformation is discussed. Beside the influence of the alloy components on the $M_{\mathrm{S}}$, the residual stress, internal defects or precipitates could change the transformation parameters.

\section{Conclusions}

The high sensitivity of the PAC method allowed us to study on the microscopic scale the martensitic phase transformation in TiPd intermetallic compounds. The hfi parameters determined experimentally were confirmed by the $a b$ initio calculations. The measured concentration dependence of the EFGs, showed that the electric field gradient corresponding to the probes substituting $\mathrm{Pd}$ atom rise with the amount of the third element, while that corresponding to probes substituting $\mathrm{Ti}$ atoms did not change significantly. The transformation parameters $M_{\mathrm{S}}$ and $T_{\mathrm{H}}$ were determined with high accuracy for wide concentration range of $\mathrm{Hf}$, In, and $\mathrm{Zr}$ admixtures. The temperature $M_{\mathrm{S}}$ of $\mathrm{TiPd}$ phase was found to decrease with increasing content of the third element, simultaneously with increase of the width $T_{\mathrm{H}}$. Moreover, the strength of these relationships depended on the type of admixture. Experimental results showed that the transformation temperature in TiPd could be tuned between $590 \mathrm{~K}$ and $810 \mathrm{~K}$ by a suitable adjustment of the ternary element content.

\section{References}

[1] K. Otsuka, X. Ren, Prog. Mater. Sci. 50, 511 (2005).

[2] S.F. Hsieh, S.K. Wu, J. Alloy. Comp. 266, 276 (1998).

[3] K.H. Eckelmeyer, Scr. Metall. 10, 667 (1976).

[4] D.R. Angst, P.E. Thoma, M.Y. Kao, J. Phys. IV (France) C8, 747 (1995).

[5] H.C. Donkersloot, J.H.N. van Vucht, J. LessCommon Met. 20, 83 (1970).

[6] A.E. Dwight, R.A. Conner, Jr., J.W. Downey, Acta Crystallogr. 18, 835 (1965).

[7] P. Villars, L.D. Calvert, Pearson's Handbook of Crystallographic Data for Intermetallic Phases, ASM, Materials Park, Ohio 1996.

[8] M. Uhrmacher, K. Pampus, F.J. Bergmaeister, D. Purschke, K.P. Lieb, Nucl. Instrum. Methods Phys. Res. B 9, 234 (1985).

[9] G. Schatz, A. Weidinger, Nuclear Condensed Matter Physics, Wiley, West Sussex, England 1996.

[10] H. Frauenfelder, R.M. Steffen, in: Perturbed Angular Correlations, Eds. K. Karlsson, E. Matthias, K. Siegbahn, North Holland, Amsterdam 1963.

[11] J. Kajfosz, Institute of Nuclear Physics (Kraków), Report No. 858/PM, 1973.

[12] T. Butz, A. Lerf, Phys. Lett. A 97, 217 (1983).

[13] P. Herzog, K. Freitag, M. Reuschenbach, H. Walitzki, Z. Phys. A 294, 13 (1980).

[14] A. Kulińska, P. Wodniecki, M. Uhrmacher, J. Alloys Comp. 494, 17 (2010).

[15] A. Kulinska, P. Wodniecki, Intermetallics 15, 1190 (2007).

[16] J. Belošević-Čavor, V. Koteski, J. Radković, Solid State Commun. 152, 1072 (2012).

[17] H. Barfuß, G. Böhnlein, H. Hohenstein, W. Kreische, M. Meinhold, H. Niedrig, Phys. Lett. A 79, 252 (1980).

[18] T. Kawamura, R. Tachi, T. Inamura, H. Hosoda, K. Wakashima, K. Hamada, S. Miyazaki, Mater. Sci. Eng. A 438-440, 383 (2006).

[19] H. Hosoda, K. Enami, A. Kamio, K. Inoue, J. Intell. Mater. Syst. Struct. 7, 312 (1996).

[20] K. Otsuka, X. Ren, Intermetallics 7, 511 (1999). 